\title{
WHY ARE SLIP LENGTHS SO LARGE IN CARBON NANOTUBES?
}

\author{
TIM G. MYERS
}

\begin{abstract}
The enhanced flow in carbon nanotubes is explained using a mathematical model that includes a depletion layer with reduced viscosity near the wall. In the limit of large tubes the model predicts no noticeable enhancement. For smaller tubes the model predicts enhancement that increases as the radius decreases. An analogy between the reduced viscosity and slip-length models shows that the term slip-length is misleading and that on surfaces which are smooth at the nanoscale it may be thought of as a length-scale associated with the size of the depletion region and viscosity ratio. The model therefore provides a physical interpretation of the classical Navier slip condition and explains why 'slip-lengths' may be greater than the tube radius.
\end{abstract}

\section{INTRODUCTION}

The classical model for flow in a circular cylindrical pipe is described by the Hagen-Poiseuille equation

$$
u_{H P}=-\frac{p_{z} R^{4}}{4 \mu}\left(1-\frac{r^{2}}{R^{2}}\right)
$$

where $u_{H P}(r)$ is the velocity in the $z$ direction, $p_{z}$ is the pressure gradient along the pipe, $R$ is the radius and $\mu$ the fluid viscosity. The corresponding flux is given by

$$
Q_{H P}=2 \pi \int_{0}^{R} r u_{H P} d r=-\frac{\pi R^{4} p_{z}}{8 \mu} .
$$

In carbon nano-tubes (CNT) it is well documented that the flow is enhanced and the true value of the flux is significantly higher than this classical value. A popular approach to explain this enhancement is to introduce a slip-length into the mathematical model, that is, the no-slip boundary condition $u(R)=0$ is replaced by

$$
u(R)=-\left.L_{s} \frac{\partial u}{\partial r}\right|_{r=R}
$$

where $L_{s}$ is the slip-length. This leads to modified velocity and flux expressions

$$
u_{\text {slip }}=-\frac{R^{2} p_{z}}{4 \mu_{1}}\left[1-\frac{r^{2}}{R^{2}}+\frac{2 L_{s}}{R}\right]_{1} \quad Q_{\text {slip }}=Q_{H P}\left(1+\frac{4 L_{s}}{R}\right),
$$


hence any magnitude of enhancement can be accounted for by using an appropriate value for $L_{s}$.

The concept of slip is an old one, attributed to Navier [1, 2, 3], and has many practical uses. When dealing with flow over a rough surface it permits a boundary condition to be applied on a flat surface, then the slip-length is related to the roughness height. In thin film theory it removes the stress singularity at the moving contact line, where the fluid front moves over a dry surface. It is also frequently invoked in studies of non-Newtonian fluids [2, 3, 4, 5, 6, 7]. In all cases the slip length is significantly smaller than the thickness of the bulk flow [1]. For example, Trethaway and Meinhart [8] carry out experiments on water flow in a coated microchannel of width $30 \mu \mathrm{m}$. Using particle imaging velocimetry they determine a slip length of $1 \mu \mathrm{m}$. In 1-2 $\mu \mathrm{m}$ channels Choi et al [9] determine values of the order $30 \mathrm{~nm}$. Molecular dynamics (MD) simulations indicate lengths of " 1 to a few nm" [10]. Whilst there is no theory to predict the slip length for a liquid flowing past a solid, there is one for gases. In this case the slip length is the mean free path of the gas [2] (for water this is $0.3 \mathrm{~nm}$ [11]). Yet, when analysing flow in CNTs, in order to sufficiently enhance the flow, the slip length must be set significantly higher than the bulk flow thickness. For example Whitby et al [12] quote lengths of 30-40 nm for experiments in pipes of $20 \mathrm{~nm}$ radius. Holt et al [11] and Majumder et al [13] quote slip lengths on the order of microns for their experiments with nanometer size pores.

Of course the high values of slip-length have led some authors to question the validity of the slip modified Hagen-Poiseuille model $[14,15]$. Cottin-Bizonne et al [16] state that the slip-length should have a single value independent of the tube radius and much less than those quoted in the literature. They attribute some of the high experimental values to contamination by hydrophobic particles. It has been proposed that the apparent slip is probably due to a decrease in the fluid viscosity near the wall caused, for example, by enhanced nucleation of dissolved gas or the formation of vapour $[1,17,16]$. Experiments have conclusively shown the existence of depletion layers between water and hydrophobic surfaces and this motivated the MD simulations of Joseph et al [18]. In keeping with this mechanism for apparent slip, Majumder et al [13] suggested that their observed flow enhancement could be attributed to an essentially frictionless interface at the CNT wall. Ruckenstein and Rajora [19] propose a gas gap at the interface between the solid and the liquid. Clearly this gap or depletion layer must be small. Experiments and simulations have shown that the fluid viscosity is in close agreement with its bulk value down to separations of about 10 molecular diameters [1]. MD simulations indicate that the fluid viscosity close to a solid boundary is position dependent [20]. For CNTs the fluid properties typically vary within an annular region approximately $0.7 \mathrm{~nm}$ from the CNT wall [18, 14, 21].

Consequently, in the following work we will investigate a mathematical model for flow including a region of low viscosity near the tube wall. In their review 
paper Mattia and Gogotsi [22] state that the question whether the nonslip boundary condition is applicable at very small scales remains open. We will provide a possible answer to this question by showing that the reduced viscosity model is analogous to a slip model, although perhaps the term 'slip length' is misleading. The slip length is typically considered to be the length-scale over which the fluid layer slips over the boundary. If instead it is interpreted as a length-scale associated with the reduced viscosity then the required high values have a more physically sensible interpretation.

\section{Mathematical Model}

Consider a pipe of cross-section $\mathrm{R}$, occupied by two fluids. In the bulk flow region, defined by $0 \leq r \leq \alpha$, we impose a viscosity $\mu_{1}$. In the annular region near the wall, defined by $\alpha \leq r \leq R$, we impose a viscosity $\mu_{2}<\mu_{1}$. Of course there is uncertainty about the values to choose for viscosity and $\alpha$. If we define the position of the transition $\alpha=R-\delta$ then, based on previous studies of water in CNTs $[14,18,21]$ in all calculations we will set $\delta=0.7 \mathrm{~nm}$. However, experiments show that the slip length increases with hydrophobicity $[9,16]$ and so for other fluids and tubes the value of $\delta$ may differ. MD simulations indicate $\mu_{2}$ varies with position [20], however, for simplicity we will take it as constant and so use an average value in the annulus. Again the constant will be system dependent.

For unidirectional pressure driven flow the appropriate mathematical model is

(5) $\frac{\mu_{1}}{r} \frac{\partial}{\partial r}\left(r \frac{\partial u_{1}}{\partial r}\right)=\frac{\partial p}{\partial z} \quad 0 \leq r \leq \alpha, \quad \frac{\mu_{2}}{r} \frac{\partial}{\partial r}\left(r \frac{\partial u_{2}}{\partial r}\right)=\frac{\partial p}{\partial z} \quad \alpha \leq r \leq R$.

Appropriate boundary conditions are

$$
\left.\frac{\partial u_{1}}{\partial r}\right|_{r=0}=0 \quad u_{2}(R, z)=0,
$$

which represent symmetry at the centreline and no-slip at the solid boundary. At the interface between the fluids, $r=\alpha$, there is continuity of velocity and shear stress

$$
u_{1}=u_{2} \quad \mu_{1} \frac{\partial u_{1}}{\partial r}=\mu_{2} \frac{\partial u_{2}}{\partial r} .
$$

The appropriate velocity expressions are then

$$
u_{1}=\frac{p_{z}}{4 \mu_{1}}\left(r^{2}-\alpha^{2}\right)-\frac{p_{z}}{4 \mu_{2}}\left(R^{2}-\alpha^{2}\right) \quad u_{2}=\frac{p_{z}}{4 \mu_{2}}\left(r^{2}-R^{2}\right) .
$$

The flux $Q_{\mu}$ is defined as the sum of fluxes in the two regions

$$
\begin{aligned}
Q_{\mu} & =2 \pi\left(\int_{0}^{\alpha} r u_{1} d r+\int_{\alpha}^{R} r u_{2} d r\right) \\
& =-\frac{\pi \alpha^{4} p_{z}}{8 \mu_{1}}\left[1-\frac{2 \mu_{1}}{\mu_{2}}\left(1-\frac{R^{2}}{\alpha^{2}}\right)\right]-\frac{\pi \alpha^{4} p_{z}}{8 \mu_{2}}\left(1-\frac{R^{2}}{\alpha^{2}}\right)^{2} .
\end{aligned}
$$


This may be related to the standard expression by

$$
Q_{\mu}=Q_{H P} \frac{\alpha^{4}}{R^{4}}\left[1+\frac{\mu_{1}}{\mu_{2}}\left(\frac{R^{4}}{\alpha^{4}}-1\right)\right] .
$$

The flow rate enhancement is defined as

$$
\epsilon_{\mu}=\frac{Q_{\mu}}{Q_{H P}}=\frac{\alpha^{4}}{R^{4}}+\frac{\mu_{1}}{\mu_{2}}\left(1-\frac{\alpha^{4}}{R^{4}}\right) .
$$

For the slip model the corresponding enhancement is

$$
\epsilon_{\text {slip }}=1+\frac{4 L_{s}}{R}
$$

In the limits $\alpha \rightarrow R$ and $L_{s} \rightarrow 0$ the expressions for $Q_{\mu}, Q_{\text {slip }}$ reduce to $Q_{H P}$ and so $\epsilon_{\mu}, \epsilon_{\text {slip }} \rightarrow 1$. If the flow rate is to be increased then $\epsilon>1$. The above expressions indicate that this requires $\mu_{1}>\mu_{2}$ or $L_{s}>0$.

To verify whether this model gives reasonable results we consider the experiments of Whitby et al [12]. Their flow enhancement indicates a slip length of $30-40 \mathrm{~nm}$ for pipes of radius $20 \mathrm{~nm}$. Setting $L_{s}=35 \mathrm{~nm}, R=20 \mathrm{~nm}$ determines their enhancement factor as $\epsilon_{\text {slip }}=8$. Rearranging the expression for $\epsilon_{\mu}$ gives

$$
\mu_{2}=\mu_{1}\left[\frac{R^{4}-\alpha^{4}}{\epsilon_{\mu} R^{4}-\alpha^{4}}\right] \text {. }
$$

To obtain the same enhancement we set $\epsilon_{\mu}=8$ and also take $\alpha=R-\delta=19.3 \mathrm{~nm}$ to find $\mu_{2}=0.018 \mu_{1}$. So, the current model will provide an enhancement factor of 8 with an average viscosity in the depletion layer approximately 0.02 times that of the bulk flow. It is interesting to note that the viscosity of oxygen is also approximately 0.02 that of water, so this value supports the depletion layer theory.

To clarify the behaviour of the current model we set $\alpha=R-\delta$. Noting that $\epsilon_{\mu}$ is simply a quartic in $\alpha$ we may expand and rearrange the expression to find

$$
\epsilon_{\mu}=1+\frac{4 \delta}{R}\left(\frac{\mu_{1}}{\mu_{2}}-1\right)\left[1-\frac{3}{2} \frac{\delta}{R}+\left(\frac{\delta}{R}\right)^{2}-\frac{1}{4}\left(\frac{\delta}{R}\right)^{3}\right],
$$

which is a monotonically decreasing function of $R$. This is in accordance with the findings of Thomas \& McGaughey [14] that the enhancement factor decreases with increasing tube radius. Noting that the reduced viscosity model requires two distinct regions, hence $R \geq \delta$, the limit to the enhancement predicted by the current theory is determined by setting $R=\delta, \mu_{2} / \mu_{1}=0.018$ and $\delta=0.7 \mathrm{~nm}$ to give $\epsilon_{\mu} \approx 50$ (Whitby et al predict an enhancement of up to 45 times theoretical predictions).

Equation (15) also allows us to make further inference about the model behaviour and it's relation to the slip model. If we compare the above expression 
with that for $\epsilon_{\text {slip }}$ we may define the slip length in terms of the thickness of the depletion layer and the viscosity ratio

$$
L_{s}=\delta\left(\frac{\mu_{1}}{\mu_{2}}-1\right)\left[1-\frac{3}{2} \frac{\delta}{R}+\left(\frac{\delta}{R}\right)^{2}-\frac{1}{4}\left(\frac{\delta}{R}\right)^{3}\right]
$$

Further, noting that $\mu_{1} / \mu_{2} \gg 1$, we can identify three distinct regimes:

(1) For sufficiently wide tubes, such that $\delta / R \ll \mu_{2} / \mu_{1}$, then $\epsilon_{\mu} \approx 1$. There is no noticeable flow enhancement and the no-slip boundary condition will be sufficient.

(2) For moderate tubes, such that $(\delta / R)\left(\mu_{1} / \mu_{2}\right)$ is order 1 but $\delta / R \ll 1$ then only the leading order term of $L_{s}$ applies and

$$
\epsilon_{\mu} \approx 1+\frac{4 \delta}{R}\left(\frac{\mu_{1}}{\mu_{2}}-1\right)
$$

(3) For very small tubes where $\delta / R$ is order 1 then the full expression for $\epsilon_{\mu}$ is required.

To be specific, the no-slip Hagen-Poiseuille model is only appropriate when $\delta / R \ll$ $\mu_{2} / \mu_{1}$. If we neglect the viscosity terms in the first regime, the error in the enhancement factor would be below $5 \%$ when $4 \delta \mu_{1} /\left(R \mu_{2}\right)<0.05$. With $\mu_{2} / \mu_{1}=$ 0.018 and $\delta=0.7 \mathrm{~nm}$ this requires $R>3 \mu \mathrm{m}$. In the second regime the error would be below $5 \%$ for $3 \delta /(2 R)<0.05$ or $R>21 \mathrm{~nm}$. In this regime the apparent slip length $L_{s} \approx 39 \mathrm{~nm}$ is approximately constant, as suggested by Cottin-Bizonne et al [16] and in keeping with a number of research groups who find slip-lengths in the range 20-40 $\mathrm{nm}$ for a wide range of length-scales $[9,12,14,16]$. For $R<21 \mathrm{~nm}$ the slip length will decrease as the radius decreases.

\section{Conclusions}

The motivation behind this paper was to explain the unrealistically large sliplengths reported in nanotubes. The mathematical model developed shows that the flow enhancement can be plausibly related to a reduced viscosity model, where the viscosity in the depletion region is always much lower than in the bulk. Theoretically, in pipes with a radius greater than the depletion layer thickness, it appears that the flow enhancement can only be enhanced by an order of magnitude (not orders as reported in some papers).

In answer to the question posed in the title "why are slip-lengths so large in carbon nanotubes?" we note that there is a clear analogy between the enhancement factor for the reduced viscosity model developed in this paper and that using a slip length. This indicates that the term 'slip length' is rather misleading. The usual physical interpretation is that the slip length represents a length-scale over which the fluid slips and consequently when this exceeds the dimension of the fluid it appears physically unrealistic. In fact it appears to be a length-scale proportional to the product of the viscosity ratio and the width of the depletion 
region. This length-scale is a property of the fluid-solid system and remains approximately constant, down to very small radius tubes, and so can easily exceed the tube radius.

In a wider context the reduced viscosity model provides one possible explanation for the Navier slip boundary condition when the solid is smooth down to the nanoscale (and hence an explanation for flow enhancement). In other systems there may well be different mechanisms to explain the slip boundary condition, for example on rough surfaces one would expect the slip length to be determined by the roughness height-scale. A number of other factors will affect the flow, such as surface composition and hydrophobicity, shear rate, fluid composition etc. To a certain extent these could be incorporated into the definition of the depletion layer and average viscosity but clearly this must be linked to detailed experimental work which is beyond the scope of this theoretical study.

\section{REFERENCES}

[1] Neto C., Evans D.R., Bonaccurso E., Butt H-J, Craig V.S.J. Boundary slip in Newtonian liquids: a review of experimental studies Rep. Prog. Phys. 68 (2005), 2859-2897.

[2] White F.M. Viscous Fluid Flow. McGraw-Hill.

[3] Matthews and Hill J.M. Nanofluidics and the Navier boundary condition. Int. J. Nanotechnol., Vol. 5, Nos. 2/3, 2008.

[4] Bhushan B. (ed.) Springer handbook of nanotechnology. Springer.

[5] Myers T.G. Application of non-Newtonian models to thin film flow. Phys. Rev. E $\mathbf{7 2}$ (2005), 066302.

[6] Myers T.G. Modeling laminar sheet flow over rough surfaces. Water Resources Res. 38 (2002); DOI: 10.1029/2000WR000154.

[7] Myers T.G. Thin films with high surface tension. SIAM Rev. 40(3) (1998), 441-462.

[8] Tretheway D.C. and Meinhart C.D. Apparent fluid slip at hydrophobic microchannel walls. Phys. Fluids 14(3) (2002), L9-12.

[9] Choi C-H, Westin J.A. and Breuer K.S. Apparent slip flows in hydrophilic and hydrophobic microchannels. Phys. Fluids 15(10) (2003), 2897-2902.

[10] Qian T., Wang X-P, Sheng P. Power-Law Slip Profile of the Moving Contact Line in TwoPhase Immiscible Flows. Phys. Rev. Lett. 93(9) (2004);

DOI: 10.1103/PhysRevLett.93.094501.

[11] Holt J.K., Park H.G., Wang Y., Stadermann M., Artyukhin A.B., Grigoropoulos C.P., Noy A., Bakajin O. Fast Mass Transport Through Sub-2-Nanometer Carbon Nanotubes. Science 312 (2006), 1034; DOI: 10.1126/science.1126298.

[12] Whitby M., Cagnon L., Thanou M. and Quirke N. Enhanced Fluid Flow through Nanoscale Carbon Pipes. Nano Letters 8(9) (2008), 2632-2637.

[13] Majumder M., Chopra N., Andrews R., Hinds B.J. Enhanced flow in carbon nanotubes. Nature 438 (2005), 44. 2005

[14] Thomas J.A. and McGaughey A.J.H. Reassessing Fast Water Transport Through Carbon Nanotubes. Nano Letters 8(9) (2008), 2788-2793.

[15] Verweij H., Schillo M.C., and Li J. Fast Mass Transport Through Carbon Nanotube Membranes. Small 3(12) (2007), 1996-2004; DOI: 10.1002/smll.200700368.

[16] Cottin-Bizonne C., Cross B., Steinberger A. and Charlaix E. Boundary Slip on Smooth Hydrophobic Surfaces: Intrinsic Effects and Possible Artifacts. Phys. Rev. Lett. 94 (2005), 56-102. 
[17] Alexeyev A.A. and Vinogradova O.I. Flow of a liquid in a nonuniformly hydrophobized capillary Colloids and Surfaces A: Physicochem. and Engng Aspects 108 (1996), 173-179.

[18] Joseph S. and Aluru N. R. Why Are Carbon Nanotubes Fast Transporters of Water? Nano Letters 8(2) (2008), 452-458.

[19] Ruckenstein E. and Rajora P. On the no-slip boundary condition of hydrodynamics. J. Coll. Interf. Sci. 96(2) (1983), 488-491.

[20] Pozhar L.A. Structure and dynamics of nanofluids: Theory and simulations to calculate viscosity. Phys. Rev. E 61(2) (2000), 1432-1446.

[21] Thomas J.A. and McGaughey A.J.H. Density, distribution, and orientation of water molecules inside and outside carbon nanotubes. J. Chem. Phys. 128 (2008), 084715; DOI: $10.1063 / 1.2837297$.

[22] Mattia D and Gogotsi Y. Review: static and dynamic behavior of liquids inside carbon nanotubes. Microfluid Nanofluid 5 (2008) 289-305; DOI 10.1007/s10404-008-0293-5.

[23] Schoch R.B. Transport phenomena in nanofluidics. Rev. Modern Phys. 80 (2008); DOI: $10.1103 /$ RevModPhys .80 .839 .

Tim G. Myers

Centre de Recerca Matemàtica

Campus de Bellaterra, Edifici C, 08193 BeLLATERRA

BARCELONA, SpAin

E-mail address: tmyers@crm.cat 